\title{
HEADACHE
}

\section{Chronic migraine linked to reduced antioxidant capacity}

New research has linked chronic migraine to reduced antioxidant capacity, providing support for the idea that supplementation with antioxidants—-such as riboflavin and coenzyme Q10-might benefit some patients with migraine.

"Chronic migraine is a highly disabling condition, but the pathogenesis of migraine and the mechanisms of migraine chronification are poorly understood," says Cinzia Lucchesi, who led the study. Some previous studies have reported increased levels of markers of oxidative

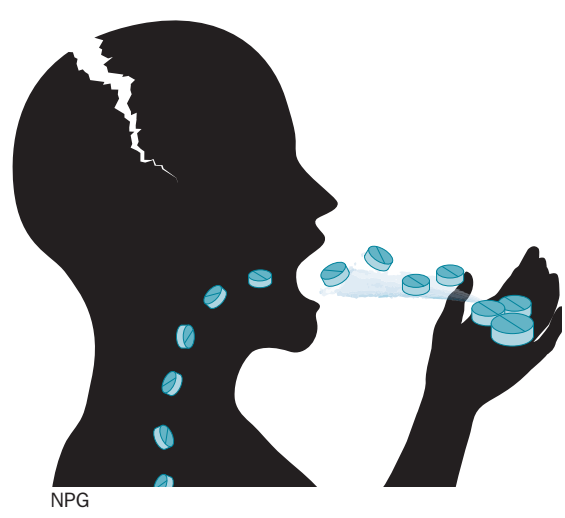

stress in patients with migraine, which supports the hypothesis that impaired oxidative mitochondrial metabolism could be involved in migraine pathogenesis.

Lucchesi and colleagues assessed the plasmatic levels of ferric-reducing antioxidant power and total thiol groups-markers of antioxidant capacity-in 33 patients with chronic migraine and medication-overuse headache $(\mathrm{CM}+\mathrm{MOH})$, and in 33 control participants.

In patients with $\mathrm{CM}+\mathrm{MOH}$, biomarkers of antioxidant power were reduced to about $50-60 \%$ of the levels seen in healthy controls. The reduced antioxidant capacity is unlikely to be related to medication, because no differences were detected between patients overusing NSAIDs and patients overusing triptans.

Next, the investigators plan to extend the study by evaluating biomarkers of antioxidant capacity in different patient groups, such as patients with episodic migraine. Lucchesi notes that the underpinnings of increased oxidative stress in migraine remain elusive. "Further investigation is certainly necessary to define whether the role of increased oxidative stress in migraine is causal or consequential."

A few small trials have reported riboflavin and coenzyme Q10 to reduce migraine attacks in some patients, but more double-blinded trials with longer follow-up times are needed to confirm the finding.

One of the studies reported that the benefit was influenced by the patient's mitochondrial haplotype. In future, identification of individuals who are particularly vulnerable to oxidative stress might help identify which patients would benefit from antioxidant therapy to prevent migraine.

\section{Hemi Malkki}

Original article Lucchesi, C. et al. Evidences of reduced antioxidant activity in patients with chronic migraine and medication-overuse headache. Headache doi:10.1111/ head12608 